\title{
Afrontamiento al estrés y su relación con el tipo de jornada laboral en docentes de educación primaria ${ }^{4}$
}

\author{
José Cháidez Nevárez \\ Maestro en Educación con Campo en la Práctica Educativa \\ Secretaría de Educación del Estado de Durango \\ Correo electrónico: cheper_1011@hotmail.com
}

Arturo Barraza Macías

Doctor en Ciencias de la Educación

Universidad Pedagógica de Durango

Correo electrónico: tbarraza-2017@hotmail.com
Recibido: 18/08/2017

Evaluado: 04/102017

Aceptado: 16/04/2018

\section{Resumen}

El principal objetivo de esta investigación es establecer la relación que hay entre las estrategias de afrontamiento al estrés laboral docente y el tipo de jornada laboral. Para el logro de este objetivo se llevó a cabo un estudio correlacional, transversal y no experimental a través de la aplicación de una encuesta a 61 profesores de Educación Primaria, pertenecientes a la Zona Esc. No. 40 de Canatlán y a la Zona Esc. No. 33 de Santiago Papasquiaro, ambas en el estado de Durango, México. Su principal resultado permite afirmar la no relación entre ambas variables, por lo que se considera que el trabajar en una escuela con más o menos horario laboral no determina la frecuencia de uso, ni el tipo de las estrategias de afrontamiento que utilizan los docentes para enfrentar su estrés. No obstante, es necesario continuar indagando variables contextuales para determinar su papel en el afrontamiento del estrés.

\section{Palabras clave}

Afrontamiento, estrés, adaptación, maestros y jornada laboral.

4 Para citar este artículo: Cháidez, J. \& Barraza, A. (2018). Afrontamiento al estrés y su relación con el tipo de jornada laboral en docentes de educación primaria. Informes Psicológicos, 18(2), pp. 63-75 http://dx.doi.org/10.18566/ infpsic.v18n2a04 


\section{Coping with stress and its relation with the type of working day in primary school teachers}

Abstract

This research seeks to establish the relation between coping strategies to teacher work stress and the type of workday. To do so, a correlational, cross-sectional and non-experimental study was carried out through the application of a survey to 61 Primary Education teachers belonging to the Sch. Zone No. 40 of Canatlán and the Sch. Zone. No 33 of Santiago Papasquiaro, both located in the state of Durango, Mexico. Its main result allows affirming the non-relationship between both variables. Thus, it is considered that working in a school with more or less working hours does not determine the frequency of use, nor the type of coping strategies used by teachers in order to face stress. However, it is necessary to continue investigating contextual variables in order to determine their role in coping with stress.

Keywords

\section{Confronto do estres e sua relação com o tipo de jornada laboral em docentes de educação primaria \\ Resumo}

0 principal objetivo desta pesquisa é estabelecer a relação existente entre as estratégias de confronto do estres laboral docente e 0 tipo de jornada laboral. Para a consecução deste objetivo foi feito um estudo correlacional, transversal e não experimental por meio da aplicação de uma enquete a 61 professores de educação primaria, pertencentes à zona escolar No. 40 de Canatlán e à zona escolar No. 33 de Santiago Papasquiaro, ambas do estado de Durango, México. Seu principal resultado permite propor a não relação entre as duas variáveis, pelo que é considerado que 0 trabalho em uma escola com um horário laboral maior ou menor, não é determinante para a frequência nem o tipo de estratégias de confronto usadas pelos docentes para encarar seu estrese. Porém, é preciso continuar indagando variáveis contextuais para determinar seu papel no confronto do estrese. 


\section{ntroducción}

Tradicionalmente se han investigado diversos tópicos del campo educativo, como los aprendizajes, las evaluaciones, las estrategias de enseñanza, las metodologías $\mathrm{y}$, últimamente, las competencias, entre otros temas; sin embargo, en la actualidad existe una tendencia a investigar la vida profesional de los docentes: ¿Cómo viven la docencia?, ¿Por qué se estresan?, ¿Por qué enferman?, ¿Qué sienten al interactuar con la práctica docente? etc. Bajo esta tendencia es que se realiza el presente trabajo de investigación, enfocado en las estrategias de afrontamiento que utilizan los docentes para afrontar su estrés.

\section{Antecedentes}

Las estrategias de afrontamiento al estrés han sido estudiadas en diferentes agentes educativos, como es el caso de los docentes (Carmona, Vega, Carrillo, Lozano \& Burciaga, 2015; Salas \& Barraza, 2017; Skaalvik \& Skaalvik, 2015) y los alumnos (Feliciano-López et al., 2017; Gustems-Carnicer \& Calderón, 2013; Halgravez, Salinas, Martínez \& Rodríguez, 2016).

En el caso de las investigaciones realizadas con docentes, las estrategias de afrontamiento se han estudiado principalmente con relación al estrés (Acosta \& Burguillos, 2014; Antoniou, Ploumpi \& Ntalla, 2013; Barraza \& Gracia, 2016; Gracia \& Barraza, 2014; Martínez, 2015) y el síndrome de burnout (Acosta \&
Burguillos, 2014; Barbosa, Muñoz, Rueda \& Suárez, 2009; Foley \& Murphy, 2015; Gantiva, Jaimes \& Villa, 2010; Martínez, 2015; Montero-Marin, PradoAbril, Piva, Gascon \& García-Campayo, 2014); así mismo, se han investigado con relación a variables esencialmente cognitivas, como es el caso del autoconcepto (Ibarra, Armenta \& Jacobo, 2014), autoeficacia (Gismero-González et al., 2012), inteligencia emocional percibida (Augusto-Landa, López-Zafra \& Pulido-Martos, 2011) y la evaluación de las propias emociones (Nizielski, Hallum, Schütz \& Lopes, 2013), o personales, como el nivel académico profesional docente (Contreras \& Barraza, 2016; Laguna, 2016; Salas \& Barraza, 2017; Valadez, Bravo \& Vaquero, 2011), la titularidad laboral (Gismero-González et al., 2012), el nivel salarial de los docentes (Contreras \& Barraza, 2016) o nivel de experiencia (Carton \& Fruchart, 2014).

Algunos otros estudios lo han relacionado con el bullying (Elizalde, 2010), el apoyo social (Gracia \& Barraza, 2014), quejas vocales (Zambon, Moreti \& Behlau, 2013) o técnicas de gestión del aula (Romi, Lewis \& Roache, 2013); sin embargo, como se puede observar, existe una tendencia a relacionar las estrategias de afrontamiento, como variable cognitivo-conductual, con otras variables esencialmente cognitivas o personales, por lo que existe la necesidad de explorar cómo algunas variables contextuales, como condiciones laborales, pueden influir en el uso o no de ciertas estrategias de afrontamiento.

Si bien "la mayoría de estos estudios se han centrado en el papel de determinados rasgos tradicionales de personalidad (neuroticismo, extraversión, 
optimismo, pesimismo, etc.) como predictores del afrontamiento eficaz o ineficaz" (Krzemien, 2007, p. 140), existe otra perspectiva denominada contextualista (Aldwin \& Revenson, 1987; Lazarus \& Folkman, 1986) que busca asignarle un papel más protagónico a la naturaleza de la situación, esto es, a las características contextuales.

En ese sentido, en la presente investigación se pretende estudiar la relación entre el afrontamiento al estrés laboral docente y el tipo de jornada laboral en docentes de educación primaria, que en el caso de la presente investigación puede ser de tres tipos: horario ampliado, tiempo completo y escuela regular (jornada normal). El supuesto de partida es que a mayor horario que cubrir, mayor carga de trabajo y, por ende, mayor estrés.

\section{Modelo Teórico}

Para Lázarus y Folkman (1986) el afrontamiento se constituye por "los esfuerzos cognitivos y conductuales en constante cambio para la resolución de demandas internas y/o externas específicas que son valoradas como desbordantes o excesivas para los recursos de las personas y que ponen en peligro su bienestar" (p. 141).

Por su parte, Morán, Landeros y González (1997) denominan estrategias de afrontamiento a las acciones que desarrollan los sujetos, tendientes a frenar, amortiguar y, en la medida de lo posible, anular los efectos y las consecuencias de las situaciones estresantes.
En este sentido, Krzemien (2007) clasifica el afrontamiento en tres modalidades, según a lo que esté dirigido:

- Afrontamiento cognitivo: en este tipo de afrontamiento existe el intento deliberado de encontrarle significado al suceso estresante y valorarlo de forma que resulte menos desagradable.

- Afrontamiento conductual: este afrontamiento está dirigido a confrontar la realidad del evento estresante, manejando sus consecuencias.

- Afrontamiento afectivo: en este afrontamiento se busca la regulación de los aspectos emocionales desarrollados a partir del evento estresante con la intención explícita de mantener el equilibrio afectivo.

Para la presente investigación cobra relevancia la distinción que existe entre las estrategias de afrontamiento activo, que están enfocadas a confrontar la situación estresante y que normalmente son identificadas como adaptativas, de otras que son denominadas pasivas y que consisten en el no enfrentamiento o el desarrollo de conductas de evitación, las que son consideradas desadaptativas (Brissette, Scheier, \& Carver, 2002; Dávila \& Guarino, 2001).

Más allá del tipo de estrategias de afrontamiento que se puedan utilizar, Lazarus (2000) expone la utilidad de considerar el proceso de afrontamiento en términos relacionales, es decir, donde se interceptan tanto variables personales como contextuales; esto es, las que se ubican en el entorno inmediato, 
así como las que subyacen en el interior de la persona.

Paralelo al proceso de interacción, este autor destaca el significado que el individuo construye a partir de dichas relaciones con el medio, lo cual opera a un nivel más alto de abstracción. Al respecto, afirma que la mente contempla la intersección de ambos tipos de variables y considera tanto las condiciones ambientales como las particularidades de la persona al hacer una valoración de su amenaza.

A partir de este posicionamiento teórico, la presente investigación pretende determinar la relación que hay entre las estrategias de afrontamiento y el tipo de escuela donde se trabaja, siendo ésta segunda variable la condición ambiental que se intenta indagar.

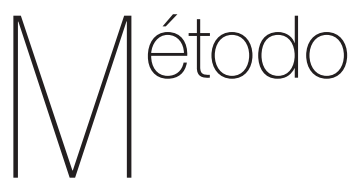

\section{Participantes}

En el presente estudio, de corte correlacional, transversal y no experimental, se contó con la participación de 61 profesores de Educación Primaria, pertenecientes a la Zona Escolar No. 40 de Canatlán y de la Zona Escolar No. 33 de Santiago Papasquiaro, ambas en el estado de Durango, México. La selección de estos profesores obedeció a la buena disposición de los mismos para participar en la investigación y a la accesibilidad geográfica y temporal para hacerles la encuesta.
La distribución de estos profesores, según las variables sociodemográficas indagadas en el background del cuestionario fue la siguiente: a) 24 son mujeres y 37 hombres; b) siete tienen estudios máximos de Normal Básica, 39 de licenciatura y 15 de maestría; c) 42 pertenecen a la Zona Escolar No. 40 de Canatlán y 19 a la Zona Escolar No. 33 de Santiago Papasquiaro; y d) 25 laboran en escuelas de jornada normal, 17 en escuelas de horario ampliado y 19 en escuelas de tiempo completo.

Los criterios de inclusión para participar en la investigación fueron el ser profesor de educación primaria, el laborar en alguna de las escuelas de cada una de las zonas escolares seleccionadas y tener una antigüedad mínima en la escuela de tres años. Este último criterio obedece a la necesidad de asegurar su inserción, con el tiempo suficiente, en estas condiciones ambientales.

\section{Técnica e instrumento}

Para recolectar la información se utilizó la técnica de la encuesta y como instrumento una versión breve del "Inventario de Estrategias de Afrontamiento del Estrés Docente" (Gracia \& Barraza, 2014). El inventario constaba originalmente de 33 ítems, pero para la presente investigación se usaron solamente 20 ítems, haciendo una selección de 10 ítems que correspondían a un afrontamiento activo o adaptativo y 10 ítems que correspondieran a un afrontamiento pasivo o desadaptativo.

Los autores, en su versión original del instrumento, no hacen una clasificación 
de las estrategias de las que se compone su inventario por lo que la selección se realizó a partir de la clasificación realizada por un panel de expertos compuesto por tres investigadores en el campo del estrés. A cada uno de ellos se les presentó dos veces el instrumento (uno para identificar las estrategias de afrontamiento de carácter adaptativo y otro para identificar las estrategias de afrontamiento de carácter desadaptativo; en cada caso se les pidió calificaran cada ítem en base a cuatro posibles respuestas: no pertenece, probablemente no pertenece, probablemente si pertenece y si pertenece. A partir de sus respuestas, se obtuvo la media y se seleccionaron las 10 estrategias para cada caso.

Esta versión breve presentó una confiabilidad en alfa de Cronbach de .76 y una confiabilidad por mitades de .75 según la fórmula de Spearman-Brown para muestras iguales. Se obtuvo además evidencias de validez a partir del procedimiento denominado análisis de consistencia interna, encontrando que todos los ítems, salvo el número cuatro, se correlacionan de manera positiva, con un nivel de significación menor a .05 , con la media general obtenida por cada encuestado, siendo el valor $r$ menor de .254 y el mayor de .553.

Esta versión breve del inventario se responde con un escalamiento de seis valores, donde cero (0) es nunca y cinco (5) es siempre. Este cuestionario se complementó con tres variables sociodemográficas que constituyeron el background: sexo, nivel máximo de estudio y tipo de escuela.
En la aplicación de este instrumento se tomaron en cuenta una serie de recomendaciones que están relacionadas con ciertas condiciones éticas que deben tenerse en cuenta a fin de facilitar la recolección de datos: a) el anonimato en los cuestionarios, b) el planteamiento de preguntas sobre las variables sociodemográficas de carácter muy general para evitar que los docentes encuestados se sintieran incómodos al contestar, y c) que en la presentación del cuestionario estuviera visible y en un primer plano la razón social de la Institución Educativa que respaldaba dicho cuestionario. Así mismo, se redactó una presentación donde se les solicitó su consentimiento, se les aseguró la confidencialidad de las respuestas y se les recordó que estaban en libertad de negarse a responderlo si así lo decidían.

\section{Procedimiento}

El cuestionario fue se aplicó durante el mes de mayo del año 2016. El análisis de resultados se realizó en tres momentos con el Programa SPSS, versión 23. En un primer momento se realizó el análisis de las propiedades psicométricas del instrumento a partir de los estadísticos Alfa de Cronbach, confiabilidad por mitades según la fórmula de Spearman-Brown y $r$ de Pearson; en un segundo momento se obtuvo la estadística descriptiva usando como medida de tendencia central la media aritmética; y en un tercer momento se hizo el análisis correlacional a través del estadístico $r$ de Pearson. En todos los análisis correlacionales la regla de decisión fue $p<.05$. 


\section{Resultados}

Los datos descriptivos obtenidos en el análisis del inventario aplicado se presentan en la tabla 1. Como se muestra las estrategias de afrontamiento que más utilizan los docentes encuestados son "Busco alternativas para solucionar las situaciones que me estresan", "Planifico y organizo las actividades que tengo que realizar" y "Pienso de manera positiva"; mientras que las que menos utilizan son "Me desahogo gritando o llorando", "Leo la biblia" y "Asisto con un psicólogo".

Tabla1.

Datos descriptivos de los ítems que conforman el inventario aplicado

\begin{tabular}{|c|c|c|}
\hline Items & Media & Desviación estándar \\
\hline 1. Escucho música & 3.61 & 1.295 \\
\hline 2. Busco alternativas para solucionar las situaciones que me estresan & 3.82 & 1.310 \\
\hline 3. Leo la biblia & .97 & 1.505 \\
\hline 4. Me desahogo gritando o llorando & .77 & 1.203 \\
\hline 5. Acostumbro pedir sugerencias a mis compañeros & 2.62 & 1.604 \\
\hline 6. Administro bien mi tiempo & 3.34 & 1.365 \\
\hline 7. Dejo la situación que me estresa en manos de Dios & 1.49 & 1.831 \\
\hline 8. Me dedico a descansar & 2.28 & 1.473 \\
\hline 9. Salgo de paseo con mis amigos & 2.10 & 1.609 \\
\hline $\begin{array}{l}\text { 10. Trato de pensar en otra cosa agradable que no tenga que } \\
\text { ver con la situación que me provoca estrés }\end{array}$ & 3.33 & 1.502 \\
\hline 11. Establezco prioridades y trato los problemas en base a ellas & 3.46 & 1.373 \\
\hline 12. No pienso en lo que me estresa & 2.56 & 1.587 \\
\hline 13. Analizo y reflexiono sobre lo que me provoca el estrés & 3.33 & 1.524 \\
\hline 14. Rezo alguna oración & 2.10 & 1.930 \\
\hline 15. Procuro distraerme haciendo otras cosas (actividades manuales, bailar, leer, etc.) & 3.25 & 1.748 \\
\hline 16. Planifico y organizo las actividades que tengo que realizar & 3.89 & 1.266 \\
\hline $\begin{array}{l}\text { Trato de concentrarme y hacer todo lo posible por } \\
\text { desprenderme de esa energía negativa que me invade }\end{array}$ & 3.80 & 1.152 \\
\hline 18. Asisto con un psicólogo & .26 & .794 \\
\hline 19. Leo sobre lo que me estresa y aplico las soluciones propuestas & 2.46 & 1.555 \\
\hline 20. Pienso de manera positiva & 4.11 & 1.226 \\
\hline
\end{tabular}

Nota: las medias más altas se destacan con negritas y las más bajas con cursivas 
En su conjunto, las estrategias de afrontamiento de carácter activo (media de 4.5) son más utilizadas que las de carácter pasivo (media de 4.10). Con relación al análisis correlacional, tras comprobar la normalidad de los datos (nivel de significación de K-S= .200) se realizó un análisis correlacional con el estadístico $r$ de Pearson (Tabla 2) mostrando los resultados que el tipo de jornada laboral no se relaciona con las estrategias de afrontamiento al estrés en los docentes de educación primaria.

Tabla 2.

Resultados del análisis correlacional entre estrategias de afrontamiento y tipo de jornada laboral

\begin{tabular}{|c|c|}
\hline Items & $r$ \\
\hline 1. Escucho música & .070 \\
\hline 2. Busco alternativas para solucionar las situaciones que me estresan & -.001 \\
\hline 3. Leo la biblia & -.068 \\
\hline 4. Me desahogo gritando o llorando & -.071 \\
\hline 5. Acostumbro pedir sugerencias a mis compañeros & -.113 \\
\hline 6. Administro bien mi tiempo & .104 \\
\hline 7. Dejo la situación que me estresa en manos de Dios & -.028 \\
\hline 8. Me dedico a descansar & -.075 \\
\hline 9. Salgo de paseo con mis amigos & .049 \\
\hline $\begin{array}{l}\text { 10. Trato de pensar en otra cosa agradable que no tenga que } \\
\text { ver con la situación que me provoca estrés }\end{array}$ & -.115 \\
\hline 11. Establezco prioridades y trato los problemas en base a ellas & .078 \\
\hline 12. No pienso en lo que me estresa & -.046 \\
\hline 13. Analizo y reflexiono sobre lo que me provoca el estrés & .177 \\
\hline 14. Rezo alguna oración & .025 \\
\hline 15. Procuro distraerme haciendo otras cosas (actividades manuales, bailar, leer, etc.) & -.177 \\
\hline 16. Planifico y organizo las actividades que tengo que realizar & .061 \\
\hline $\begin{array}{l}\text { 17. Trato de concentrarme y hacer todo lo posible por desprenderme } \\
\text { de esa energía negativa que me invade }\end{array}$ & .067 \\
\hline 18. Asisto con un psicólogo & .014 \\
\hline 19. Leo sobre lo que me estresa y aplico las soluciones propuestas & .039 \\
\hline 20. Pienso de manera positiva & -.066 \\
\hline
\end{tabular}

Nota: ${ }^{*} p<.05$

En el caso del tipo de estrategias de afrontamiento utilizadas para afrontar el estrés y su relación con el tipo de jornada laboral, los resultados marcan un valor $r$ de .007 para las estrategias activas y de
.023 para las estrategias pasivas, ambos valores con niveles de significación superiores a .05, por lo que se puede afirmar la no relación. 
La focalización en variables personales, de índole cognitiva, ha sido una constante cuando se estudia el estrés. Desde el sustento teórico hegemónico centrado en la valoración cognitiva de Lazarus y Folkman (1986) hasta sus potenciales variables predictoras, como sería el caso de la indefensión aprendida (Barraza, 2011) o la autoeficacia (Merino \& Lucas, 2015), por mencionar solo algunas.

Esta misma tendencia se observa con las estrategias de afrontamiento y los mismo se investiga con variables como motivación autodeterrminada (Oliveira et al., 2016) o como autoestima (Larrota, Sánchez \& Sánchez, 2016), por poner solo algunos ejemplos. Contra esta tendencia se alzan varias voces que han dado lugar a la perspectiva contextualista bajo la cual se inscribió el objetivo de la presente investigación. Siguiendo la recomendación de Lázarus (2000) se exploró la relación entre las estrategias de afrontamiento y el entorno laboral inmediato de los docentes, siendo un precedente el estudio de Gismero-González et al., (2012) que estudian la titularidad del centro (tipo de sostenimiento: privado vs. público) y el nivel educativo (Infantil, Primaria, Secundaria, Bachillerato y Formación Profesional)

En este estudio, Gismero-González et al., (2012) indican que los profesores de centros con sostenimiento público utilizan con más frecuencia las estrategias de Pasividad Pesimista, y Autorreferencia Obsesiva, que los profesores de centros con sostenimiento privados. Por su parte los profesores de Infantil y Primaria emplean más la Planificación Racional ante los problemas de conducta que presentan sus alumnos, y menos la Pasividad Pesimista ante la sobrecarga de trabajo, que los profesores de Secundaria.

En el caso de la presente investigación los resultados obtenidos indican la no relación entre las variables estudiadas por lo que se considera que el trabajar en una escuela con más o menos horario laboral no determina la frecuencia de uso ni el tipo de las estrategias de afrontamiento que utilizan los docentes para enfrentar su estrés. Cabe destacar, con relación a estos resultados, que no se localizaron estudios previos similares con los cuales se pueda hacer una contrastación por lo que se considera necesario no descartar su estudio para futuras investigaciones.

En lo general, y no obstante este resultado, los autores siguen considerando, al igual que Lazarus (2000), que las variables ambientales tienen, y pueden jugar, un papel relevante en el uso de las estrategias de afrontamiento por lo que se hace necesario seguir con la indagación al respecto.

Por otra parte, el que las estrategias de afrontamiento que más se usen sean de carácter adaptativo y que, en lo general, también sean éstas las que se usen con mayor frecuencia, indica una tendencia de los docentes encuestados a enfrentar el problema que les aqueja y les genera estrés; problema que normalmente se encuentra también en su ámbito laboral, lo que coincide con Álvarez (2014), Muñoz y Correa (2012), Contreras y Barraza (2016) y Gantiva et al., (2010), salvo en el caso de las estrategias relacionadas con la religión en esta última investigación. El 
que los docentes encuestados utilicen preferentemente este tipo de estrategias indica una mayor realización personal en el trabajo y, por lo tanto, menores niveles de estrés (Martínez, 2015).

La principal limitación de este estudio es la poca muestra de docentes encuestados y la distribución desigual con relación a la variable tipo de escuela por lo que se recomienda seguir indagando al respecto y tomar los presentes resultados como indicativos.

\section{Referencias}

Acosta. M. \& Burguillos, A. (2014). Estrés y burnout en profesores de primaria y secundaria de Huelva: las estrategias de afrontamiento como factor de protección. International Journal of Developmental and Educational Psychology/INFAD: Revista de Psicología (IJODAEP), 4(1), 303-309. doi: 10.17060/ijodaep.2014.n1.v4616.

Aldwin, C. M. \& Revenson, T. A. (1987). Does coping help? A reexamination of the relation between coping and mental health. Journal of Personality and Social Psychology, 53(2), 337-348. Recuperado de https://www. researchgate.net/profile/Carolyn_Aldwin/ publication/19535402_Does_Coping_ Help_A_Reexamination_of_the_Relation_ Between_Coping_and_Mental_Health/ links/00b7d52f27fcb2c209000000/DoesCoping-Help-A-Reexamination-of-theRelation-Between-Coping-and-MentalHealth.pdf
Álvarez, M.V. (2014). Estrés laboral, síndrome de Burnout y técnicas de afrontamiento en docentes de educación general básica de un colegio privado de Quito en el periodo Marzo a Julio del 2013. (Tesis de grado, Universidad Internacional SEK). Recuperada de http://repositorio.uisek. edu.ec/bitstream/123456789/894/1/ Ver\%C3\%B3nica\%20Alvarez\%20V..pdf

Augusto-Landa, J.M., López-Zafra, E. \& PulidoMartos, M. (2011). Inteligencia Emocional Percibida y estrategias de afrontamiento al estrés en profesores de enseñanza primaria: propuesta de un modelo explicativo con ecuaciones estructurales (SEM). Revista de Psicología Social, 26(3), 413-425. doi: 10.1174/021347411797361310

Antoniou, A.S., Ploumpi, A. \& Ntalla, M. (2013). Occupational stress and professional burnout in teachers of primary and secondary education: the role of coping strategies. Psychology, 4(3), 349-355. doi:10.4236/psych.2013.43A051

Barbosa, L.C., Muñoz, M.L., Rueda, P.X. \& Suárez, K.G. (2009). Síndrome de burnout y estrategias de afrontamiento en docentes universitarios. Revista Iberoamericana de Psicología: Ciencia y Tecnología, 2(1), 21-30. Recuperado de http://revistas. iberoamericana.edu.co/index.php/ ripsicologia/article/view/174/597

Barraza, A. (2011). Indefensión Aprendida y su relación con el nivel de estrés autopercibido en alumnos de Licenciatura. EnA. Barraza \& A. Jaik (coord.). Estrés, Burnout y bienestar subjetivo. Investigaciones sobre la salud mental de los agentes educativos (34-51), Durango: Red Durango de Investigadores Educativos 
Barraza, A. \& Gracia, A. (2016). Las estrategias de afrontamiento proactivo ante el estrés generado por la reforma educativa. En J.B. Miranda, A. Barraza y A. Galván (eds.), De la educación superior a la administración municipal Un recuento de experiencias heurísticas (pp. 98-110). México: ReDIE.

Brissette, I., Scheier, M. F., \& Carver, C. S. (2002). The role of optimism in social network development, coping and psychological adjustment during a life transition. Journal of Personality and Social Psychology, 82(1), 102-111. doi: 10.1037//0022-3514.82.1.102

Carmona, L.S., Vega, N., Carrillo, E., Lozano, D.I. \& Burciaga, J.H. (2015). Estilos de afrontamiento de profesoras expuestas a violencia de género. Revista Electrónica de Psicología Iztacala, 18(4), 1747-1762. Recuperado de http://www.medigraphic. com/pdfs/epsicologia/epi-2015/epi154v. pdf

Carton, A. \& Fruchart, E. (2014). Sources of stress, coping strategies, emotional experience: effects of the level of experience in primary school teachers in France. Educational Review, 66(2), 245-262. doi:10. 1080/00131911.2013.769937

Contreras, P. \& Barraza, A. (2016). Estrategias de afrontamiento al estrés laboral en docentes de educación básica. Eureka, 13(2), 248-262.

Dávila, B., \& Guarino, L. (2001). Fuentes de estrés y estrategias de afrontamiento en escolares venezolanos. Revista Interamericana de Psicología, 35(1), 97-112. Recuperado de https://www. researchgate.net/profile/Leticia_Guarino/ publication/26610700_Fuentes_de_ Estres_y_Estrategias_de_Afrontamiento_ en_Escolares_y_Venezolanos/ links/54d4e8b50cf2970e4e63de7b/ Fuentes-de-Estres-y-Estrategiasde-Afrontamiento-en-Escolares-yVenezolanos.pdf?origin=publication_detail

Elizalde, A. (2010). Estudio descriptivo de las estrategias de afrontamiento del bullying, en profesorado mexicano. Electronic Journal of Research in Educational Psychology, 8(20), 353-372. Recuperado de http:// www.redalyc.org/pdf/2931/293121995017. pdf

Feliciano-López, V., Ortega, J.M., JiménezChafey, M.I., Duarté-Vélez, Y., ChárriezCordero, M. \& Bernal, G. (2017). Exposición y conocimiento sobre la prevención del suicidio, estigma, estrategias de afrontamiento y recursos en estudiantes de nuevo ingreso de la Universidad de Puerto Rico, Recinto de Río Piedras. Revista Puertorriqueña de Psicología, 28(1), 46-61.

Foley, C. \& Murphy, M. (2015). Burnout in Irish teachers: Investigating the role of individual differences, work environment and coping factors. Teaching and Teacher Education, 50, 46-55. doi:10.1016/j.tate.2015.05.001

Gantiva, C.A., Jaimes, S. \& Villa, M.C. (2010). Síndrome de burnout y estrategias de afrontamiento en docentes de primaria y bachillerato. Psicología desde el Caribe, 26, 36-50. Recuperado de http://www. redalyc.org/articulo.oa?id=21319039003.

Gismero-González, M.E., Bermejo, L., Prieto, M., Cagigal, V., García-Mina, A. \& Hernández, V. (2012). Estrategias de Afrontamiento Cognitivo, Auto-eficacia y variables laborales. Orientaciones para prevenir el Estrés Docente. Acción Psicológica, 9(2), 87-96. doi: 10.5944/ ap.9.2.4107 
Gracia, A. \& Barraza, A. (2014). Estresores organizacionales, estrategias de afrontamiento y apoyo social en docentes de educación primaria. México: IUNAES.

Gustems-Carnicer, J. \& Calderón, C. (2013). Coping strategies and psychological wellbeing among teacher education students. European Journal of Psychology of Education, 28(4), 1127-1140. doi:10.1007/ s1021

Halgravez, L.A., Salinas, J.E., Martínez, G.I. \& Rodríguez, O.E. (2016). Percepción de estrés en universitarios, su impacto en el desempeño académico, afrontamiento y apoyo familiar. Revista Mexicana de Estomatología, 3(2), 27-36.

Ibarra, E., Armenta, M. \& Jacobo, H.M. (2014). Autoconcepto, estrategias de afrontamiento y desempeño docente profesional. Estudio comparativo en profesores que trabajan en contextos adversos. Profesorado. Revista de Currículum y Formación de Profesorado, 18(1), 223-239. Recuperado de http://www. redalyc.org/pdf/567/56730662013.pdf

Krzemien, D. (2007). Estilos de Personalidad y Afrontamiento Situacional Frente al Envejecimiento en la Mujer. Revista Interamericana de Psicología, 41(2), 139150. Recuperado de http://www.psicorip. org/Resumos/PerP/RIP/RIP041a5/ RIP04115.pdf

Laguna, J.L. (2016). Respuestadeafrontamiento y nivel académico profesional docente en instituciones educativas, Trujillo - 2016. UCV - Scientia, 8(2), 141-147. Recuperado de http://revistas.ucv.edu.pe/index.php/ UCV-SCIENTIA/article/view/1034/809

Larrota, R., Sánchez, L. \& Sánchez, J. (2016). Niveles de autoestima y uso de estrategias de afrontamiento en un grupo de personas privadas de la libertad en un centro de reclusión de la ciudad de Bucaramanga/ Colombia. Informes Psicológicos, 16(1), 5164. doi: 10.18566/nfpsicv16n1a03

Lazarus, R. S. \& Folkman, S. (1986). Estrés y procesos cognitivos. Barcelona, España: Martínez Roca.

Lazarus, R. S. (2000). Estrés y emoción. Manejo e implicaciones en nuestra salud. Bilbao, España: Desclée de Brouwer.

Martínez, J.P. (2015). Cómo se defiende el profesorado de secundaria del estrés: burnout y estrategias de afrontamiento. Revista de Psicología del Trabajo y de las Organizaciones, 31(1), 1-9. doi: 10.1016/j. rpto.2015.02.001

Merino, E. \& Lucas, S. (2015). La autoeficacia y la autorregulación como variables moderadoras del estrés laboral en docentes de educación primaria. Universitas Psychologicas, 15(1), 205-218. Recuperado de http://www.scielo.org.co/ pdf/rups/v15n1/v15n1a16.pdf

Montero-Marin, J., Prado-Abril, J., Piva, M.M., Gascon, S. \& García-Campayo, J. (2014). Coping with stress and types of burnout: explanatory power of different coping strategies. PLOS ONE 9(2): e89090. doi:10.1371/journal.pone.0089090

Morán, C., Landeros, R. \& González, M.T. (1997). COPE-28: un análisis psicométrico de la versión en español del Brief COPE. Universitas Psychologica, 9(2), 543-552.

Muñoz, C.F. \& Correa, C.M. (2012). Burnout docente y estrategias de afrontamiento en docentes de primaria y secundaria. Revista Colombiana de Ciencias Sociales, 
3(2), 226-242. Recuperado de http://www. funlam.edu.co/revistas/index.php/RCCS/ article/view/887/836

Nizielski, S., Hallum, S., Schütz, A., \& Lopes, P.N. (2013). A note on emotion appraisal and burnout: The mediating role of antecedentfocused coping strategies. Journal of Occupational Health Psychology, 18(3), 363-369. doi:10.1037/a0033043

Oliveira, L.P., Nascimento, J.R., Vissoci, J.R., Ferreira, L., Silva, P. \& Vieira, J.L. (2016). Motivación autodeterminada y estrategias de afrontamiento en futbolistas: un estudio con jugadores en diferentes fases de desarrollo deportivo. Revista de Psicología del Deporte, 25(2), 261-269. Recuperado de https://ddd.uab.cat/pub/revpsidep/ revpsidep_a2016v25n2/revpsidep_ a2016v25n2p261.pdf

Romi, S., Lewis, R. \& Roache, J. (2013). Classroom management and teachers' coping strategies: Inside classrooms in Australia, China and Israel. Prospects, 43(2), 215-231. doi: 10.1007/s11125-013-9271-0
Salas, O. \& Barraza, A. (2017). Estrategias de afrontamiento proactivo al estrés laboral que utilizan los maestros de educación primaria. En A. Barraza y V. Gutiérrez (coord.). El estudio de la realidad educativa a partir de sus actores (pp. 59-70). México: IUNAES.

Skaalvik, E.M. \& Skaalvik, S. (2015). Job satisfaction, stress and coping strategies in the teaching profession-what do teachers say? International Education Studies, 8(3), 181-192. doi:10.5539/ies.v8n3p181

Valadez, A., Bravo, M.C. \& Vaquero, J.E. (2011). Estrategias de afrontamiento empleadas por docentes universitarios. Revista Electrónica de Psicología Iztacala, 14(1), 65-76. Recuperado de http://www. revistas.unam.mx/index.php/repi/article/ view/24794/23323

Zambon, F., Moreti, F. \& Behlau, M. (2013). Coping strategies in teachers with vocal complaint. Journal of Voice, 28(3), 341-348. doi:10.1016/j.jvoice.2013.11.008 